\title{
High Precision, Rapid Readout of Cryogenic Temperature Sensors in the Space Shuttle Environment
}

\author{
CHRISTOPHER E. WOODHOUSE, MEMBER, IEEE
}

\begin{abstract}
A compact and rugged system to perform 4-wire determination of resistance of germanium resistance thermometers (GRT) near $1 \mathrm{~K}$ to 1 part in 65 thousand in $1 \mathrm{~s}$ using $20-\mathrm{nW}$ excitation is described. Constraints unique to cryogenic and aerospace systems are addressed. A microcomputer-controlled, hybrid analog/digital design is presented. Test results are presented and future developments are discussed.
\end{abstract}

\section{INTRODUCTION}

$\mathrm{T}$ HE USE of supercooled cryogens to cool detectors on space-based observatories is becoming increasingly important. Temperatures required range from $4.4 \mathrm{~K}$ down to $0.1 \mathrm{~K}$ and below. Correct calibration of the spacecraft detectors requires precise knowledge of the absolute temperature and even more precise measurement of relative temperatures. Measurement of these temperatures in-situ for monitoring and control presents some special problems to the design engineer.

In most cases, the points from which temperatures are to be measured are inside a specially constructed vacuum vessel known as a dewar. Such a dewar will contain an amount of cryogen, such as liquid helium. A telescope within the dewar will typically view space through the end that is opened after launch. The vessel is usually filled before launch with several hundred liters of helium at 1.4 $\mathrm{K}$ and sealed. Special venting systems allow boiled off gas to escape to the vacuum of space. The lifetime of the scientific package is usually limited by the time that the cryogen lasts. Projects are underway to demonstrate the possibility of refilling the dewar on orbit [1], [2], [9]. Nevertheless, the desire to minimize the amount of heat that leaks into the dewar or that which is dissipated inside is paramount. Precise temperature monitoring is also important in the diagnosis of heat leakage paths.

Temperatures are sensed using commercially available devices known as germanium resistance thermometers (GRT's); see Fig. 1 and, for example, [3]. The GRT is a small chip of lightly doped germanium which exhibits a resistance versus temperature curve roughly proportional

Manuscript received April 25, 1989

The author was with the NASA/Goddard Space Flight Center, Greenbelt, MD. He is now with John Hopkins School of Medicine, Baltimore, MD 21205.

IEEE Log Number 8932863.
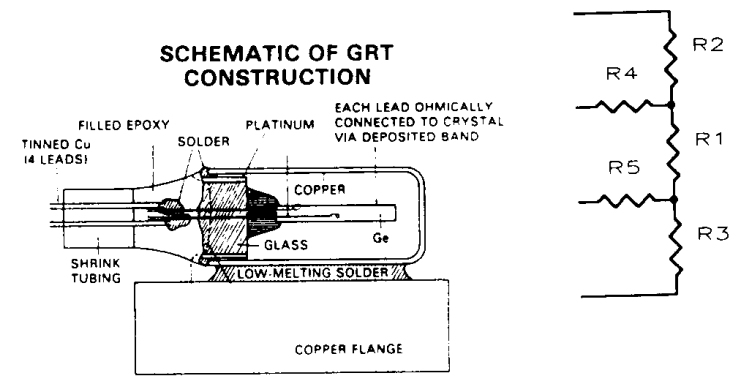

Fig. 1. GRT and equivalent circuit.

to $1 / t^{5}$ over limited ranges. For our application, the resistance varies from $300 \Omega$ to $20 \mathrm{k} \Omega$. The GRT is sensed using the 4-wire technique. That is, current is supplied through two end terminals and voltage is sensed across two middle terminals. See Fig. 1 for equivalent model and note the parasitic components.

The small size of the GRT enhances its speed of response. A small hole is drilled in the case of the sensor to allow liquid helium to bathe the sensing element directly. This will decrease the temperature errors due to self-heating. Very low excitation currents will also reduce this effect, in addition to reducing the power dissipated inside the dewar. Voltages within the dewar must be kept low in some applications due to the close proximity of the temperature sensors to the optical detectors. Crosstalk is often a problem. Currents of $1 \mu \mathrm{A}$ rms or less are used which with a resistance of $20 \mathrm{k} \Omega$ results in less than 20 nW dissipation.

A typical application will have many GRT's at various locations within the dewar. Since no practical system for multiplexing the signals at $2 \mathrm{~K}$ has been developed, each of the sensors must have 4 wires from the cold area to the outside of the dewar. This is a temperature differential of around $300^{\circ} \mathrm{C}$ and represents a large heat load on the dewar. GRT's are wired with 36 AWG manganin wire from the cold to warm areas. These manganin wires have a large thermal resistance and a manageable electrical resistance (30-300 $\Omega$ ). Large temperature differentials give rise to unpredictable and large dc offsets at dissimilar metal junctions.

Shielded cables will typically carry the signals from the dewar to the electronics box situated on the spacecraft. 
The total cable distance may be quite long. In one current application, the cables are about $25 \mathrm{ft}$ long.

The absolute accuracy required by this design is 1.0 $\mathrm{mK}$ over a range from 1.15 to $4.4 \mathrm{~K}$. The stability and resolution is required to be better than $100 \mu \mathrm{K}$. This corresponds to a resistance resolution on the order of 1 part per 32.5 thousand. Most applications in this area are required to read temperatures from a suite of sensors in a timely fashion. A baseline for this design is about $1 \mathrm{~s}$ per reading. In spite of low signal levels and the noise environment, the required resolution is a realistic number.

The electronics suitable for flight aboard the shuttle must be robust and insensitive to temperature extremes. The ambient temperature limits for the design presented here are $-45^{\circ} \mathrm{C}-+70^{\circ} \mathrm{C}$. The system must be able to withstand the appropriate vibrational loads. The parts used must be of high reliability because of the high cost of failure. Electronic parts must not release gasses and must operate in a vacuum. These facts generally limit the components available to the circuit designer.

\section{Design (System Architecture)}

\section{A. Sensing Strategy}

The strategy chosen to sense the GRT's is illustrated in Fig. 2. The basic technique is to use a digital simulation of a classic synchronous demodulator to coherently detect the voltage returned from a sensor that is excited by a sinusoidal, $1-\mu \mathrm{A}$ current.

$\mathrm{AC}$ excitation was chosen for a number of reasons. Junction potentials and high gain operational amplifier configurations tend to exhibit a phenomenon known as "flicker," " $1 / f$," or "pink," noise [5]. The terminology derives from the observation that the amplitude of this noise grows larger as the frequency of interest decreases. It is this noise that fundamentally limits the usefulness of a dc system.

This means that integration of this noise for longer periods does not reduce its intensity. The point at which the $1 / f$ noise amplitude drops below the "white" or broadband noise in the Precision Monolithics, Inc. OP-27A amplifier used in this circuit is about $2.7 \mathrm{~Hz}$ [4]. This frequency is known as the " $1 / f$ corner." A frequency of 18.7 $\mathrm{Hz}$ was chosen to be safely above this limit. The upper limit on the excitation frequency is determined by the allowable effect of capacitive parasitics, mostly in the cable system. Certain spacecraft applications require specific frequency allocation to avoid crosstalk with sensitive detectors.

\section{B. Sine Wave Generator}

A 16-bit read only memory (ROM)/digital-to-analog converter (DAC) combination was chosen as the sine wave generator. This was chosen over an analog, amplitude stabilized oscillator to gain precise knowledge of timing, simplicity, and robustness. The resulting 4-chip set is very compact and requires no adjustment. The reference voltage for this converter is derived from the analog-to-digital converter (ADC) described later. The hybrid systems DAC chosen (HS3160) settles within $1 \mu$ s to its assigned value. Given an $18.7-\mathrm{Hz}$ rate, and 2048 samples/cycle, the sample time is $26 \mu \mathrm{s} /$ sample. Deglitching, or removal of code switching transients was considered unnecessary because the high-frequency nature of the transients causes them to be filtered out by the bandwidth of the current source and the capacitance of the cable system.

\section{Current Source}

A balanced, differential current source (Fig. 3) derived from the Howland configuration [6] is used. The compliance of this current source by design is as high as possible and has been determined to be over $13 \mathrm{G} \Omega$ in the present implementation. Precision resistor networks from Electrofilms, Inc. are the key components responsible for the excellent compliance characteristics of this source. Since balanced portions of the feedback network are fabricated on a single substrate and laser trimmed to 0.05 -percent accuracy, temperature coefficients of the critical resistor ratios are reduced to very low levels. The balanced nature of the output keeps systematic common mode effects to a minimum. Vishay VHS102K ultrastable metal-film resistors provide the required accuracy.

\section{Multiplexing}

A bank of analog multiplexers serve to distribute the current excitation to and return the voltage signal from one of 32 GRT sensors. The multiplexers are low leakage $(0.3 \mathrm{nA})$ complementary metal oxide semiconductor (CMOS) devices.

\section{E. Cable System}

The cable system represents up to $25 \mathrm{ft}$ of 24 AWG TFE Teflon insulated, twisted shielded pairs with an average wire to shield capacitance of $141 \mathrm{pF} / \mathrm{m}(43 \mathrm{pF} / \mathrm{ft})$ and wire-to-wire capacitance of $89 \mathrm{pF} / \mathrm{m}(27 \mathrm{pF} / \mathrm{ft})$. The connection of shield and grounds is indicated in Fig. 4. A great deal of time has been expended in the analysis and testing of various wiring systems in several satellite projects. The maximum usable frequency of the carrier is strongly influenced by the parasitic capacitance and resistance of the wiring harness. SPICE circuit simulations and testing allowed us to derive the correction factors to extract the highest accuracy from the system.

\section{F. Low Noise Preamplifier}

The voltage signal returned from the sensor will be around $20-\mathrm{mV}$ rms maximum. This signal is amplified into a $10-\mathrm{V}$ rms signal by a classic three-amplifier instrumentation amplifier. Precision thin-film resistor networks were used to maximize CMRR and gain stability. Low noise amplifiers of the PMI OP-27 type were chosen. These amplifiers exhibit a $3 \mathrm{nV} / \sqrt{\mathrm{Hz}}$ white voltage noise level, a $1 / f$ corner at $2.7 \mathrm{~Hz}$, and a noise current of $1 \mathrm{pA} / \sqrt{\mathrm{Hz}}$ at $10 \mathrm{~Hz}$ with a current noise corner at $140 \mathrm{~Hz}$. A source resistance of $20 \mathrm{k} \Omega$ yields a current induced noise voltage of $20 \mathrm{nV} / \sqrt{\mathrm{Hz}}$. The resultant equivalent noise voltage is 


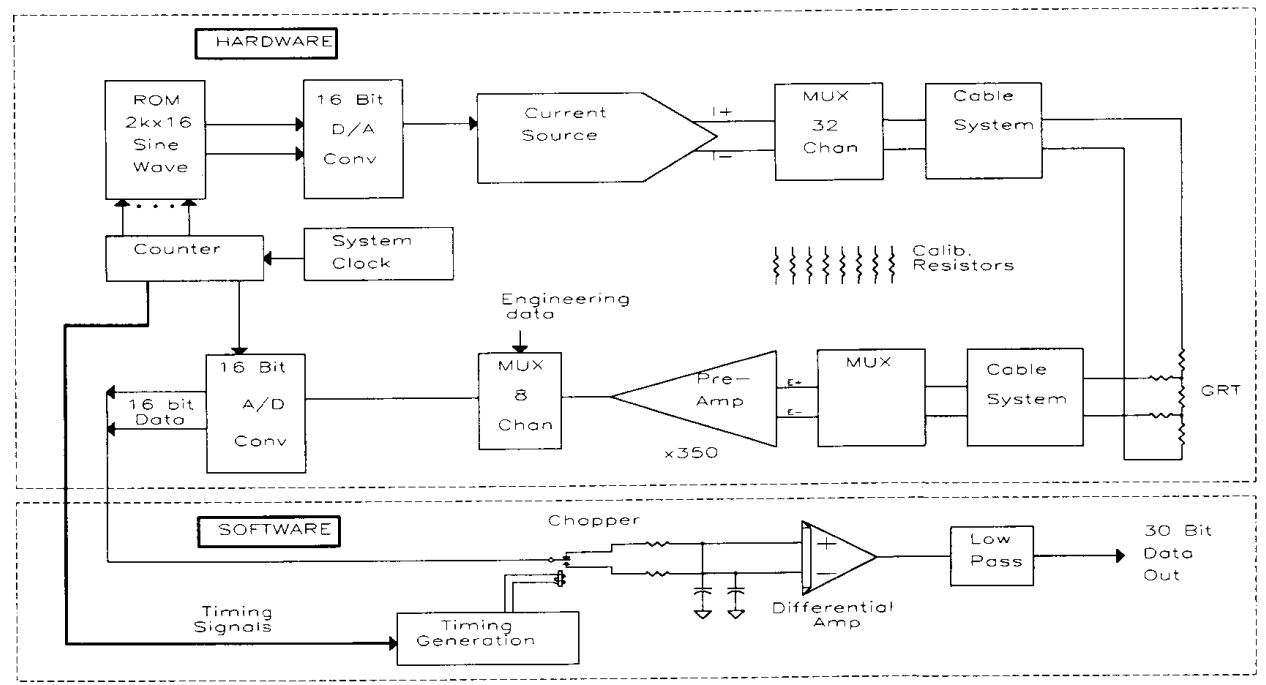

Fig. 2. Measurement strategy-note that all components in the HARDWARE dotted box are physical components while those in the SOFTWARE box are digitally simulated in the microprocessor.

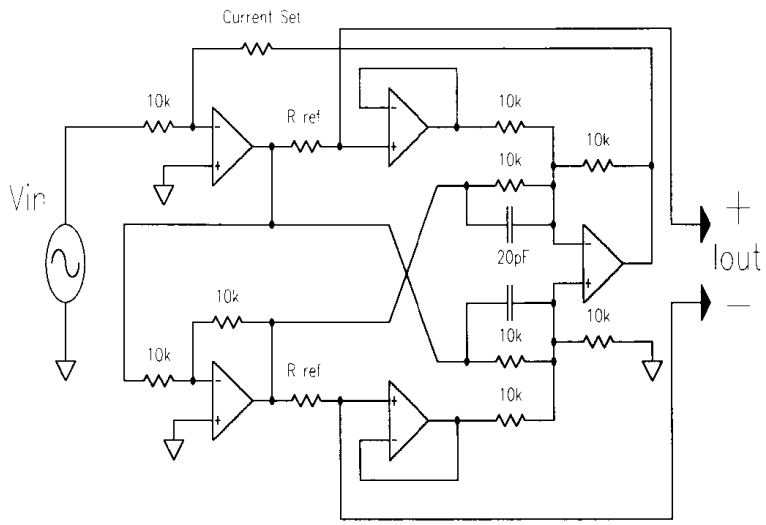

Fig. 3. Balanced, differential 1- $\mu$ A current source.

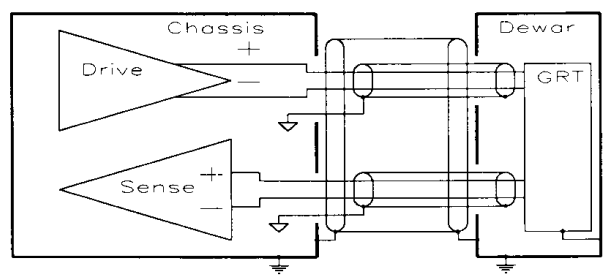

Fig. 4. Grounding and shielding.

then about $20.2 \mathrm{nV} / \sqrt{\mathrm{Hz}}$. A noise amplitude at the 16 bit level $(-96 \mathrm{~dB})$ is desirable. This corresponds to 20 $\mathrm{mV} / 65536=305-\mathrm{nV} \mathrm{rms}$. The final system bandwidth must, therefore, be less than $(305 / 20)^{2}=225 \mathrm{~Hz}$. (Note, for comparison, that a 741-type Op-Amp has a voltage noise density of $20 \mathrm{nV} / \sqrt{\mathrm{Hz}}$ and a current density of 20 $\mathrm{pA} / \sqrt{\mathrm{Hz}}$.)

The thermal noise power available from the GRT $=$

\section{$k T \Delta F$ where:}

$k \quad$ Boltzmann's constant $=1.38 \times 10^{-23} \mathrm{~W} \cdot \mathrm{s} / \mathrm{K}$, $T$ absolute temperature $=1.5 \mathrm{~K}$,

$\Delta F$ system bandwidth,

$R \quad$ sensor resistance $=30 \mathrm{k} \Omega$.

The thermal noise voltage is, therefore,

$$
E_{t}=4 k T R \Delta F=\sqrt{621 \times 10^{-21} \Delta F} .
$$

A system bandwidth of $100 \mathrm{~Hz}$ yields an rms thermal noise component of 7.9-nV rms. This is clearly negligible. Notice that with the sensor at room temperature, this noise becomes nontrivial.

The preamplifier bandwidth is set so that the following relation holds at the carrier frequency:

$$
\left|\frac{1}{1+j \omega R c}\right| \geq 1-2^{-16} ; \quad \omega=2 \pi \times 18.7 \mathrm{~Hz} .
$$

This limits the preamp bandwidth to just over $10 \mathrm{kHz}$ which presents a smooth signal to the ADC during the $50-\mu$ s conversion time.

\section{G. Analog-to-Digital Conversion}

A high reliability, qualified 16 -bit converter is used to digitize the ac signal after preamplification. The $\mathrm{MN} 5290 \mathrm{H} / \mathrm{B}$ ADC is manufactured by the Micro Networks, Inc. and is procured to /883 specifications. No sample-and-hold is used. This eliminates systematic errors introduced by the sample-and-hold. Errors due to signal voltages drifting through quantization bins during the conversion period are averaged out by the sampling technique described below. Spurious errors in excess of what is predicted by an analysis of the theoretical successive approximation technique have not been observed.

The conversion time is about $50 \mu \mathrm{s}$ and the conversion 
rate is chosen to produce exactly 512 samples per cycle of the carrier. This corresponds to a sample rate of 9.57 kilosamples/s and a sample time of $104 \mu \mathrm{s}$.

\section{H. Calibration Resistors}

A set of eight Vishay type VHS102K metal foil resistors forms an on-board reference. These resistors exhibit a temperature coefficient of less than $1 \mathrm{ppm} / \mathrm{C}$. Our temperature range of $115^{\circ} \mathrm{C}$ indicates that the calibration resistors may vary up to $115 \mathrm{ppm}$. 16-bit accuracy demands 15 -ppm accuracy. In-flight measurement of the circuit board temperature and correction of the calibration resistors will be made to achieve a 16-bit reference source.

\section{On-line Processing}

A technique for compensation of minute to minute drift of excitation amplitude and gain magnitudes will be used. Calibration resistors will be sampled at regular intervals during the course of normal operation. The entire set of eight resistors, and their temperatures, will be collected every $88 \mathrm{~s}$. That is, every 11 th reading will be of a calibration resistor. These readings will be averaged with corresponding readings from the previous $10 \mathrm{~min}$ using appropriate weightings, and will form a dynamic calibration table. A third-order polynomial curve is fit to the data and this curve forms the transfer function for the instrument. The curve is updated every time a calibration resistor is read. The computations involved are performed in the ground processing computer and take less than $100 \mathrm{~ms}$ on an IBM PC/AT class personal computer. The results from this technique are dramatic and the required accuracies over temperature and time could not be achieved without this processing.

\section{J. Digital Processor}

The subsequent processing of the digitized signal is carried out in a microprocessor system. The system chosen for flight is a conventional, minimum-mode Harris 80C86based CMOS microcomputer running at $3 \mathrm{MHz}$. The system has 16k EPROM, 16k static RAM, and the serial and parallel input and output lines required to perform the temperature measurement, a pressure measurement, liquid/vapor discrimination, and measurement of the circuit board temperature, in addition to handling commands and transmitting telemetry. Since the implementation is straightforward and conventional, this paper will not deal with the digital processor. It suffices to say that microprocessor-based systems have just recently been flown on the space shuttle by our group at NASA.

\section{K. Fault Tolerance and Redundancy}

Fault tolerance is achieved by duplicating the circuit twice in the enclosure. Normal operation is achieved with both systems operating simultaneously on their own sets of sensors. A master/slave protocol has been established and data flows out of the system through one of the two digital processing units. In the event of a single failure, the functional side of the system will continue to collect data. Mission success is assured with half of the sensors functional. There are duplicate sensors at critical locations.

\section{Measurement Algorithm}

The process whereby a sensor is measured is as follows:

1) select desired sensor coincident with zero crossing of drive signal;

2) wait one cycle for transients to settle;

3) clear summing registers-POS, NEG, $Q+$, and $Q-$;

4) do for 16 sycles;

a) read $\mathrm{A} / \mathrm{D}$ converter;

b) apply delay, if desired;

c) apply window function;

d) if phase $=0^{\circ}-180^{\circ}$ THEN POS $=$ POS + Sample ELSE NEG $=$ NEG + Sample;

e) if phase $=90^{\circ}-270^{\circ}$ THEN $Q+=Q++$ Sample ELSE $Q^{-}=Q_{-}+$Sample

5) in-phase magnitude $=$ POS - NEG;

6) quadrature result $=Q+-Q-$;

7) transmit 24-bit results via telemetry.

Note: Timing is derived from sine wave generator address inputs.

\section{A. Analysis:}

The synchronous demodulator (synchronous detector, coherent detector, suppressed carrier demodulator, or precision rectifier) implemented here provides a very narrow bandwidth detector centered over the carrier frequency. The narrow bandwidth provides rejection of the bulk of the wide-band noise expected in the Shuttle cargo bay (and EMC testing). The digital implementation of the chopper provides a number of dramatic improvements in the performance of this system over earlier analog implementations of the system. Previous systems were limited by parasitic problems in analog switches used for the chopper, settling time delays, charge injection in the averaging capacitors, offsets and gain variations in the differential amplifier, and missing codes and offsets in the ADC. The new technique eliminates most of these problems.

The first step in the analysis is to convince oneself that the errors induced by the ADC are of negligible magnitude. Sampling is taking place at $9.5 \mathrm{kHz}$. This is 256 times the Nyquist rate for the $18.7-\mathrm{Hz}$ carrier. Quantization errors may be assumed to be an additive white noise process with an amplitude around the 16th bit level [8]. Since the minimum signal, $0.3 \mathrm{mV}$, will range over alnost 1000 counts, and a typical signal may range over tens of thousands of counts, missing code errors will be independent and uncorrelated with the input signal. Wideband thermal noise referred to the input will be around 20 $\mathrm{nV} / \sqrt{\mathrm{Hz}} \times \sqrt{10 \mathrm{kHz}}=2-\mu \mathrm{V} \mathrm{rms}$. This alone will carry the ADC over 4 counts. Since the contribution of a white 


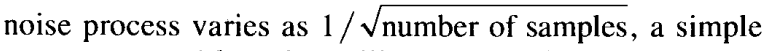
average over 16 cycles will attenuate the noise by approximately a factor of $90,7 \mathrm{bits}$, or $39 \mathrm{~dB}$. This process is reminiscent of the well-known "dithering" technique, wherein a known noise process is injected into a measurement in order to extract increased resolution from a quantizing device.

The effects of integral nonlinearity in the ADC will manifest themselves as a harmonic distortion in the digitized signal. Further analysis of this effect is underway in our laboratories.

Settling time in the instrument is eliminated by clearing the digital register at the beginning of each measurement. Since the chopper, averaging circuit, and differential amplifier are mathematical constructs and processing is carried out with full 32-bit precision, it is reasonable to expect ideal performance from these components. There is no charge injection in a simulated capacitor as there is no gain variation or offset in a simulated differential amplifier. The designer's job is much easier when the equations work without consideration of the physical device.

All real amplifiers are operating on ac signals, and therefore, show no dc offset or bias. The only dc amplifier in the system is a virtual one, and it shows none of these errors.

Incoming data throughout the measurement cycle may be weighted by appropriate functions. Bartlett, Hanning, and Hamming filters were applied to experimental data. Performance with a simple rectangular (boxcar) filter was found to be superior to any of the others. It is thought that the signal energy lost by weighting the data negated advantage in out-of-band rejection provided by the other filters tried. The boxcar filter is also easier to implement with discrete logic.

The magnitude of the Fourier transform of a rectangular filter is

$$
\begin{aligned}
&|F(\omega)|=|\sin (\omega N / 2) / \sin (\omega / 2)| \\
& \text { where } N=512 \times 16=8192 .
\end{aligned}
$$

The first null in the spectrum will occur at $2 \times \pi N=$ $\pi / 4096$ in the digital domain. This corresponds to 1.17 $\mathrm{Hz}$ in the analog domain. The digital implementation, therefore, provides a filter bandwidth of $1.16 \mathrm{~Hz}$ in ana$\log$ frequency for an integration time of 16 cycles, or a bit less than $1 \mathrm{~s}$. A first-order analog filter of this bandwidth would require 11 time constants, or over $9 \mathrm{~s}$, to settle to within 1 part in $2^{16}$.

A disadvantage of the digital implementation is that there are side lobes to the filter function. Peaks of the side lobes occur at $x=(2 n+1) \pi / N$ where $n=[1 \cdots$ $(N / 2-1)]$. The first and largest side lobe has a magnitude of 0.212 , or $-13.5 \mathrm{~dB}$. The second is down $18 \mathrm{~dB}$ and the minimum side lobe is down $-78 \mathrm{~dB}$. A Blackman window should theoretically give a $55-\mathrm{dB}$ first side lobe, but as mentioned before, this was not observed experimentally.

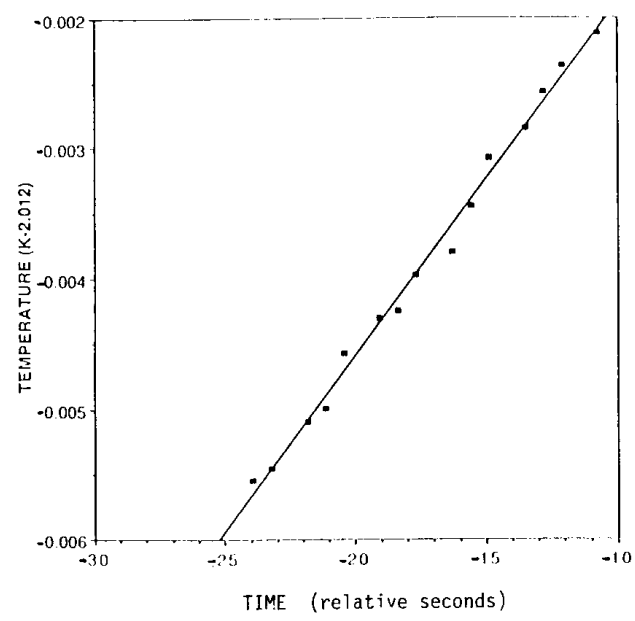

Fig. 5. Example of temperature measurement.

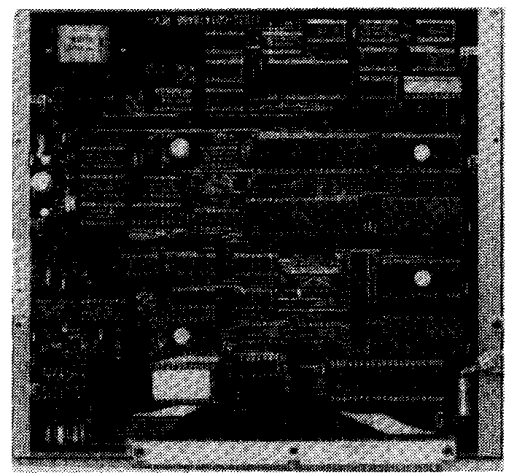

Fig. 6. Digital processor.

\section{Results}

Breadboard systems have been tested in the Goddard cryogenics laboratory. Flight system boards shown in Fig. 6-8 are undergoing testing at this time.

Measurements of the calibration resistors for 16 cycles each on board in a temperature controlled environment show a stable output count to the 18 th bit $(3 \mathrm{ppm})$.

Measurements of laboratory GRT's were performed in an unshielded, glass, supercooled dewar. Fig. 5 illustrates a recorded temperature drift. The rms scatter from the best fit line in the data shown is $0.03 \mathrm{mK}$. The peak resolution observed in our experiments was $7.7 \mu \mathrm{K}$. This occurred at the lowest temperatures where the $d R / d T$ of the sensors is at a maximum.

Application of the post-processing algorithm of Section II-A resulted in over an order of magnitude increase in stability. Since the accuracy can be no better than the stability, this processing is an integral part of the instrument design. 


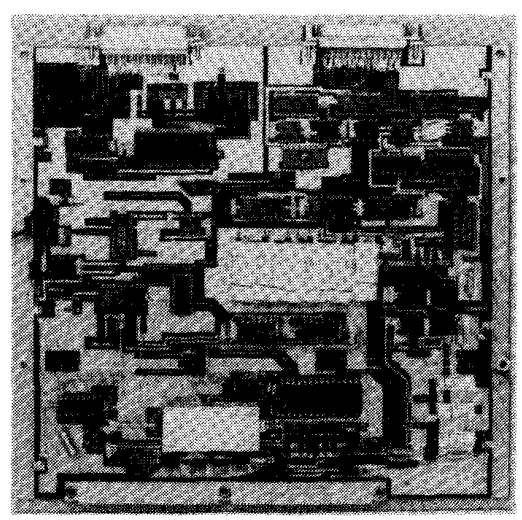

Fig. 7. Analog processor.

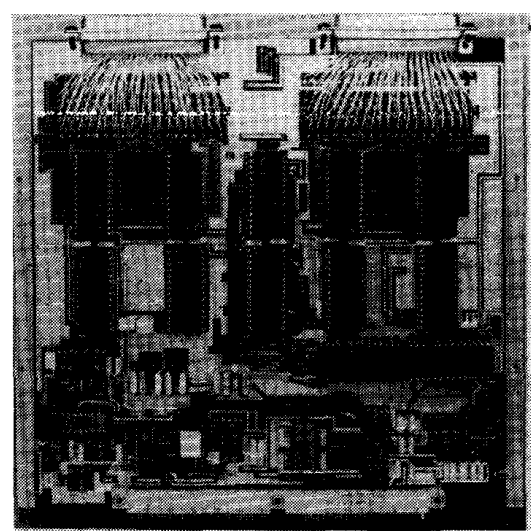

Fig. 8. Multiplexer card.

\section{Future Developments}

Future developments of this instrument will provide for higher speed of readings and greater accuracies. Appli- cations down to $0.1 \mathrm{~K}$ will demand lower excitation currents. A proposed design using 1-nA excitation is being investigated. The possibility of using pseudonoise excitation and correlation techniques will provide control of the emitted spectrum of the sensor near the detectors. Multiplexing of sensors at an intermediate stage within the dewar will reduce the size of the wiring harness and its concomitant heat load. The technique presented herein need not use a microprocessor. A hybrid analog/digital integration of the system using logic arrays and analog IC's could provide a highly compact implementation.

\section{ACKNOWLEDGMENT}

The author wishes to express thanks to the following individuals for their support in the development of this instrument: Drs. J. Sutton and M. DiPirro; D. Huff and R. Martin for design assistance; C. Caldwell and J. Pyle for branch level support; E. Zalubas, M. Chein, M. Toth, D. McDermond, A. Huffman; and Ms. A. Grant for technical assistance.

\section{REFERENCES}

[1] C. Woodhouse, A. Kashani, A. Lukemire, "Superfluid helium tanker instrumentation," IEEE Trans. Instrum. Meas., vol. 39, pp. 274-278, Feb. 1990 .

[2] M. J. DiPirro and P. Kittel, "The superfluid helium on-orbit transfer (SHOOT) flight demonstration," presented at 1987 Cryogenic Engineering Conf., St. Charles, U.S.A., 1987.

[3] "Technical specification: GR-200B," Data Sheet, Lake Shore Cryogenics, Inc.,

[4] "Linear and Conversion Products 1986/87," Precision Monolithics, Inc., Data Book, pp. 5-128, 1987.

[5] C. D. Motchenbacher and F. C. Fitchen, Low-Noise Electronic De sign. New York: Wiley, 1973.

[6] T. Henry, "Analysis and design of the op amp current source," Application Note AN-587, Motorola Semiconductor Products, Inc.

[7] R. Morrison, Grounding and Shielding Techniques in Instrumentation, 3rd ed. New York: Wiley, 1986.

[8] A. V. Oppenheim and R. W. Schafer, Digital Signal Processing. Englewood Cliffs, NJ: Prentice-Hall, 1975, ch 9, p. 404.

[9] S. R. Broen, "Liquid helium servicing from the space station," Cryogenics, vol. 28, pp. 68-76, Feb. 1988 